\title{
Comparison of QuantiFERON-TB Gold In-Tube (QFT-GIT) and tuberculin skin test (TST) for diagnosis of latent tuberculosis in haemodialysis (HD) patients: a meta-analysis of $\boldsymbol{\kappa}$ estimates
}

\author{
E. AYUBI ${ }^{1,2,3}$, A. DOOSTI-IRANI ${ }^{4}$, A. SANJARI MOGHADDAM ${ }^{5}$, \\ S. KHAZAEI ${ }^{2,4}$, K. MANSORI ${ }^{6,7}$, S. SAFIRI ${ }^{8}$, M. SANI $^{9}$ AND E. MOSTAFAVI ${ }^{10,3 *}$ \\ ${ }^{1}$ Department of Epidemiology, School of Public Health, Shahid Beheshti University of Medical Sciences, Tehran, \\ Iran \\ ${ }^{2}$ Department of Epidemiology and Biostatistics, School of Public Health, Tehran University of Medical Sciences, \\ Tehran, Iran \\ ${ }^{3}$ Department of Epidemiology and Biostatistics, Pasteur Institute of Iran, Tehran, Iran \\ ${ }^{4}$ Department of Epidemiology, School of Public Health, Hamadan University of Medical sciences, Hamadan, \\ Iran \\ ${ }^{5}$ School of Medicine, Shahid Beheshti University of Medical Sciences, Tehran, Iran \\ ${ }^{6}$ Social Determinants of Health Research Center, Kurdistan University of Medical Sciences, Sanandaj, Iran \\ ${ }^{7}$ Department of Epidemiology, School of Public Health, Iran University of Medical Sciences, Tehran, Iran \\ ${ }^{8}$ Department of Public Health, Managerial Epidemiology Research Center, School of Nursing and Midwifery, \\ Maragheh University of Medical Sciences, Maragheh, Iran \\ ${ }^{9}$ School of Medicine, Zabol University of Medical Sciences, Zabol, Iran \\ ${ }^{10}$ Research Centre for Emerging and Reemerging infectious diseases, Pasteur Institute of Iran, Tehran, Iran
}

Received 14 September 2016; Final revision 21 January 2017; Accepted 31 January 2017;

first published online 2 March 2017

\section{SUMMARY}

Diagnosis of latent tuberculosis infection (LTBI) is a concern in haemodialysis (HD) patients. Many studies have compared QuantiFERON-TB Gold In-Tube (QFT-GIT) and tuberculin skin test (TST) for detecting LTBI and reported the $\kappa$ statistic of agreement between QFT-GIT and TST in HD patients. The present study aimed to systematically review this literature and conduct meta-analysis of individual studies that estimated the $\kappa$ between QFT-GIT with TST among HD patients. All relevant published studies that were available as full-text were obtained by searching Medline (1950), Web of Sciences (1945), Scopus (1973) through May 2016. The $\kappa$ was re-estimated from the individual studies and pooled using random effect meta-analysis. Subgroup analysis and meta-regression were applied to evaluate the effect of Bacillus Calmette-Guérin (BCG) vaccination, TST cut-off points, quality of studies, sample size and age on variation of $\kappa$ estimate. Eight studies involving $901 \mathrm{HD}$ patients were included in meta-analysis. The pooled $\kappa$ estimate was $0 \cdot 28\left(I^{2}=18 \cdot 4 \%, P=0 \cdot 239,95 \%\right.$ confidence intervals $\left.0 \cdot 22-0 \cdot 34\right)$. The discordance of TST-/QFT-GIT+ was more than TST+/QFT-GIT-. History of BCG vaccination, TST cut-off points and age are related to variation of $\kappa$ estimates. TST and QFT-GIT are not comparable in detecting LTBI in HD patients. The higher TST-/QFT-GIT+ ratio compared with TST +/QFT-GIT - ratio, may indicate the superiority of QFT-GIT over TST for detection LTBI in HD patients.

\footnotetext{
* Author for correspondence: E. Mostafavi, Department of Epidemiology and Biostatistics, Pasteur Institute of Iran, Tehran, Iran and Research Centre for Emerging and Reemerging Infectious Diseases, Pasteur Institute of Iran, Tehran, Iran. (Email: mostafaviehsan@) gmail.com)
} 
Key words: Haemodialysis patients, interferon- $\gamma$ release tests, $\kappa$ statistic, meta-analysis, tuberculin skin test.

\section{INTRODUCTION}

The risk of reactivation of latent tuberculosis infection (LTBI) among haemodialysis (HD) patients is higher than in the general population [1,2], possibly due to uraemic immunodeficiency and compromised cellmediated immunity. Early detection of LTBI is important to prevent the development of active tuberculosis (TB) in the HD patients [3], and many clinical guidelines recommend screening for LTBI in HD patients.

There is no gold standard diagnostic test for LTBI. Until recently, diagnosis of LTBI was based on induration size following a tuberculin skin test (TST). This test has several limitations. False-positive results can occur following non-TB mycobacterial infection or previous Bacillus Calmette-Guérin (BCG) vaccination [4] and in people with immune deficiency the test may be falsely negative [5]. To offset these the challenges regarding TST, the interferon- $\gamma$ release assays (IGRAs) tests are introduced. IGRAs are based on the T-cells respond to restimulation with Mycobacterium tuberculosis-specific antigens by secreting interferon- $\gamma$ (IFN- $\gamma$ ) among patients with TB infection. Two such tests are QuantiFERON-TB Gold (T-SPOT.TB) and QuantiFERON-TB Gold In-Tube (QFT-GIT) [6].

Previous published study have demonstrated IGRAs have higher specificity, positive predictive values (PPV) and negative predictive value (NPV) compared with TST for LTBI detection [7]. The authors in the aforementioned study point out the results for PPV must be interpreted with caution because PPV is context dependent, but the results of NPV in low-prevalence countries are reasonably confident because the likelihood of false-negative results is low [7]. It has been suggested that in immunocompetent patients, IGRAs are superior to TST in detecting LTBI [8]. In HD patients due to impaired T-cell activation upon mitogenic stimulation, the secretion of IFN $-\gamma$ may be diminished leading to unreliable IGRAs results [9].

Many previous studies addressed the diagnostic performance of QFT-GIT and TST in HD patients and measured clinical agreement ( $\kappa$ estimate) between the tests. Reported $\kappa$ estimates range $0 \cdot 15-0 \cdot 64$ $[10,11]$. We conducted systematic review of individual studies comparing QFT-GIT with TST among HD patients and performed meta-analysis of reported $\kappa$ estimates.

\section{METHODS}

\section{Search strategy}

We searched Medline (January 1950 to May 2016), Web of Sciences (January 1945 to May 2016), and Scopus (January 1973 to May 2016). Search strategy was: 'latent tuberculosis infection', 'QuantiFERON', 'interferon-gamma release test', 'interferon-gamma release assay', 'enzyme-linked immunospot assay', 'tuberculin test', 'PPD-S', 'skin test', 'Mantoux TST', $\kappa, \kappa$-value, $\kappa$-statistic, agreement, observational study, English publishing. This search strategy was applied for search in each mentioned databases. The search strategy in detail is presented in the Supplementary. Two authors (E.A. and A.D.-I.) independently reviewed potentially relevant studies, study selection, data abstraction and quality assessment. Any disagreement between the authors was referred to other author (E.M.) for final decision. Email communication with corresponding authors of the retrieved articles was done for retrieving additional or unpublished studies or provision information on missing, incomplete and unreported variables.

\section{Study selection}

Title/abstracts of retrieved studies were independently reviewed. Full articles were obtained for review where no insufficient information was presented in the abstract. From retrieved studies in initial search as potentially relevant, original articles or letters to the editor were chosen when they have the following criteria: original data were presented for $\kappa$ and standard error (SE) estimate, QFT-GIT and TST simultaneously performed in the same individual. Studies of confirmed active TB and those screening for LTBI among KTRs (kidney transplant recipients) were excluded.

\section{Data abstraction and quality assessment}

For all included studies, the following information was extracted; first author, year of publication, study 
country, sample size, sex ratio, mean age, the history of BCG vaccination (yes/no/unknown), TST induration diameter defined as positive. The assessment of study quality was performed by application of modified STrengthening the Reporting of OBservational studies in Epidemiology (STROBE) checklist [12]. The following criteria were assessed; (1) how sample size was determined (2) the study population with inclusion and exclusion criteria were clearly defined, (3) heterogeneity of results was explained by relevant methods such as subgroup analysis, (4) appropriate methods were used to measure QFT-GIT and TST and (5) applying sensitivity analysis to clarify the effect of prevalence of the attribute. Study quality was classified as high quality - studies that fulfilled all criteria; intermediate quality - studies filing to meet at most one criterion; low quality - studies not fulfilling more than one criterion.

\section{Data analysis}

Agreement between QFT-GIT and TST is estimated as Cohen's $\kappa$. The $\kappa$ measures the degree to which agreement between two tests is greater than that expected by chance alone. SE of Cohen's $\kappa$ with $95 \%$ confidence intervals (CI) for all studies were estimated by suggested method of Fleiss JL [13]. Statistical heterogeneity across the studies was measured by $I^{2}$ test. Subgroup analysis and metaregression were used to explore the source of study heterogeneity. Publication bias was examined using funnel plots and Egger test. Analysis was performed using Stata 11.0 (Stata Corp, College Station, TX, USA).

\section{RESULTS}

\section{The characteristics of included studies}

Our search strategy yielded 6744 records. We identified 42 additional records through reference list of retrieved studies and email communication with authors - among them 10 studies fulfilled the inclusion criteria $[3,10,11,14-20]$. For two studies SE of $\kappa$ was derived from the reported confidence bounds $[19,20]$. Two studies did not include original data for $\kappa$ estimate $[10,18]$ and were not included into meta-analysis. Finally, eight studies involved a total of $901 \mathrm{HD}$ patients considered for inclusion in meta-analysis [3, 11, 14-17, 19, 20]. Figure 1 shows the selection process of the studies; and detailed

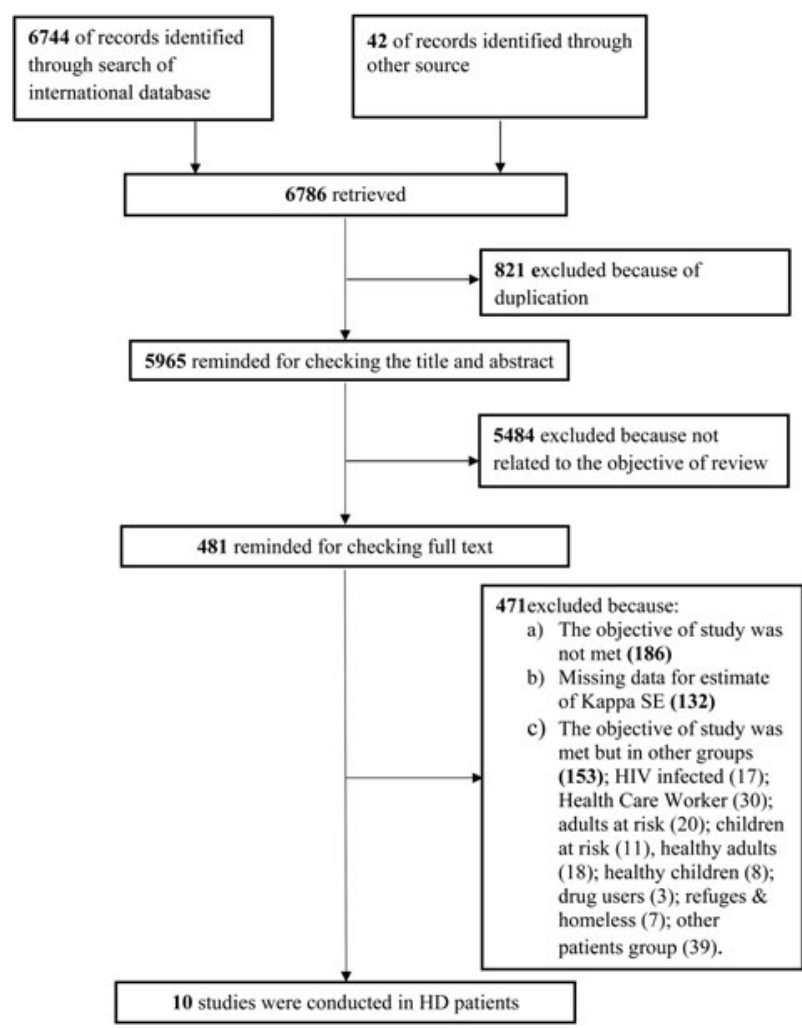

Fig. 1. A flow chart depicts the stages of retrieving references, checking eligibility criteria, and including the final articles into the meta-analysis.

information on the eight included studies is presented in Table 1 .

\section{The quality assessment and publication bias}

Four studies were of intermediate quality $[3,14,16$, 19] and four studies were low quality [11, 15, 17, 20] (Table 2). Symmetry funnel plots and Egger's test $(P$-value $=0 \cdot 11)$ suggest no publication bias (Fig. 2$)$.

\section{Statistical analysis results}

No significant heterogeneity was found among eight studies $[3,11,14-17,19,20]\left(I^{2}=18 \cdot 4 \%, P=0 \cdot 239\right)$, so the fixed-effects model was used. The pooled $\kappa$ coefficient $(95 \% \mathrm{CI})$ between TST and QFT-GIT was $0 \cdot 28(0 \cdot 22-0 \cdot 34)$ (Fig. 3).

Subgroup analysis by BCG vaccination showed that $\kappa$ coefficient $(95 \% \mathrm{CI})$ for people with and without history of BCG vaccination were $0.22(0.07-$ $0 \cdot 37)$ and $0.31(0 \cdot 14-0 \cdot 47)$, respectively. The $\kappa$ estimate for QFT-GIT and TST $(\leqslant 5 \mathrm{~mm})$, QFT-GIT and TST $(>5 \mathrm{~mm})$ and QFT-GIT and TST $(>10$ $\mathrm{mm})$ were $0.49(0 \cdot 25-0 \cdot 73), 0.28(0.22-0.34)$ and 
Table 1. Characteristics of the included studies into meta-analysis to test the clinical usefulness of QFT-GIT vs. TST for diagnosis of latent TB in HD patients

\begin{tabular}{|c|c|c|c|c|c|c|c|c|c|c|c|}
\hline First author & $\begin{array}{l}\text { Publication } \\
\text { year }\end{array}$ & Country & $\begin{array}{l}\text { Sample } \\
\text { size* }\end{array}$ & $\begin{array}{l}\text { Mean } \\
\text { of age }\end{array}$ & $\begin{array}{l}\text { Male } \\
(\%)\end{array}$ & $\begin{array}{l}\text { TST cut } \\
\text { point }\end{array}$ & $\begin{array}{l}\text { BCG } \\
\text { vaccination }\end{array}$ & $\begin{array}{l}\operatorname{TST}(+), \\
\operatorname{QFT}(+)\end{array}$ & $\begin{array}{l}\text { TST (-), } \\
\text { QFT (+) }\end{array}$ & $\begin{array}{l}\operatorname{TST}(+), \\
\operatorname{QFT}(-)\end{array}$ & $\begin{array}{l}\operatorname{TST}(-) \\
\operatorname{QFT}(-)\end{array}$ \\
\hline Seyhan EC & 2009 & Turkey & 72 & $56 \cdot 2$ & $0 \cdot 47$ & 10 & Yes & 17 & 17 & 13 & 25 \\
\hline Seyhan EC & 2009 & Turkey & 28 & 56 & - & - & No & 4 & 5 & 0 & 19 \\
\hline Ates G & 2009 & Turkey & 230 & 51.9 & $0 \cdot 50$ & 10 & Unclear & 58 & 49 & 25 & 98 \\
\hline Lee SSJ & 2010 & Taiwan & 93 & $58 \cdot 3$ & $0 \cdot 41$ & 5 & Unclear & Unclear & Unclear & Unclear & Unclear \\
\hline Lee SSJ & 2010 & Taiwan & - & - & - & 10 & Unclear & Unclear & Unclear & Unclear & Unclear \\
\hline Lee SSJ & 2010 & Taiwan & - & - & - & $15 / 10$ & Unclear & Unclear & Unclear & Unclear & Unclear \\
\hline Lee SSJ & 2010 & Taiwan & - & - & - & $18 / 10$ & Unclear & Unclear & Unclear & Unclear & Unclear \\
\hline Hoffmann M & 2010 & Switzerland & 32 & 64 & $0 \cdot 48$ & 10 & Unclear & 2 & 7 & 1 & 22 \\
\hline Hoffmann M & 2010 & Switzerland & - & - & - & 5 & Unclear & 5 & 4 & 2 & 21 \\
\hline Maden E & 2011 & Turkey & 67 & $54 \cdot 8$ & $0 \cdot 51$ & 10 & Yes & Unclear & Unclear & Unclear & Unclear \\
\hline Maden E & 2011 & Turkey & - & - & - & 5 & - & Unclear & Unclear & Unclear & Unclear \\
\hline Maden E & 2011 & Turkey & 29 & - & - & 10 & No & Unclear & Unclear & Unclear & Unclear \\
\hline Maden E & 2011 & Turkey & - & - & - & 5 & - & Unclear & Unclear & Unclear & Unclear \\
\hline Ahmadinejad Z & 2012 & Iran & 65 & $38 \cdot 5$ & $0 \cdot 59$ & 10 & Unclear & 6 & 8 & 7 & 30 \\
\hline Kim SY & 2013 & South Korea & 9 & 49 & $0 \cdot 83$ & 10 & Unclear & 4 & 2 & 0 & 3 \\
\hline Kim SY & 2013 & South Korea & 91 & 47 & $0 \cdot 55$ & 10 & Unclear & 11 & 27 & 5 & 48 \\
\hline Agrawal SK & 2015 & India & 185 & $36 \cdot 7$ & $0 \cdot 70$ & 10 & Unclear & 13 & 53 & 19 & 100 \\
\hline
\end{tabular}

* Sample size without indeterminate results. 
Table 2. Quality of studies assessing the $\kappa$ estimate between QFT-GIT and TST

\begin{tabular}{|c|c|c|c|c|c|c|}
\hline \multirow[b]{2}{*}{$\begin{array}{l}\text { Author (year of } \\
\text { publication) }\end{array}$} & \multicolumn{5}{|c|}{ Criteria* } & \multirow[b]{2}{*}{ Quality } \\
\hline & $\begin{array}{l}\text { Sample } \\
\text { size }\end{array}$ & $\begin{array}{l}\text { Study } \\
\text { participants }\end{array}$ & $\begin{array}{l}\text { Statistical } \\
\text { methods }\end{array}$ & $\begin{array}{l}\text { Un-biased } \\
\text { measurement }\end{array}$ & Prevalence & \\
\hline Ates G (2009) & + & + & + & + & - & Intermediate \\
\hline Seyhan EC (2009) & - & + & + & + & - & Low \\
\hline Hoffmann M (2010) & - & + & - & + & - & Low \\
\hline Lee SSJ (2010) & + & + & + & + & - & Intermediate \\
\hline Maden E (2011) & - & + & - & - & - & Low \\
\hline Ahmadinehad Z (2012) & - & + & - & + & - & Low \\
\hline Kim SY (2013) & + & + & + & + & - & Intermediate \\
\hline Agarwal SK (2015) & + & + & + & + & - & Intermediate \\
\hline
\end{tabular}

* Explanation of each criteria is as follows: determining study population in a logical way; using eligibility criteria and appropriate methods for participation selection; applying relevant methods to examine heterogeneity of $\kappa$ estimate across subgroups; using valid measurement methods for both QFT-GIT and TST; conducting sensitivity analysis to address the effect of HD prevalence.

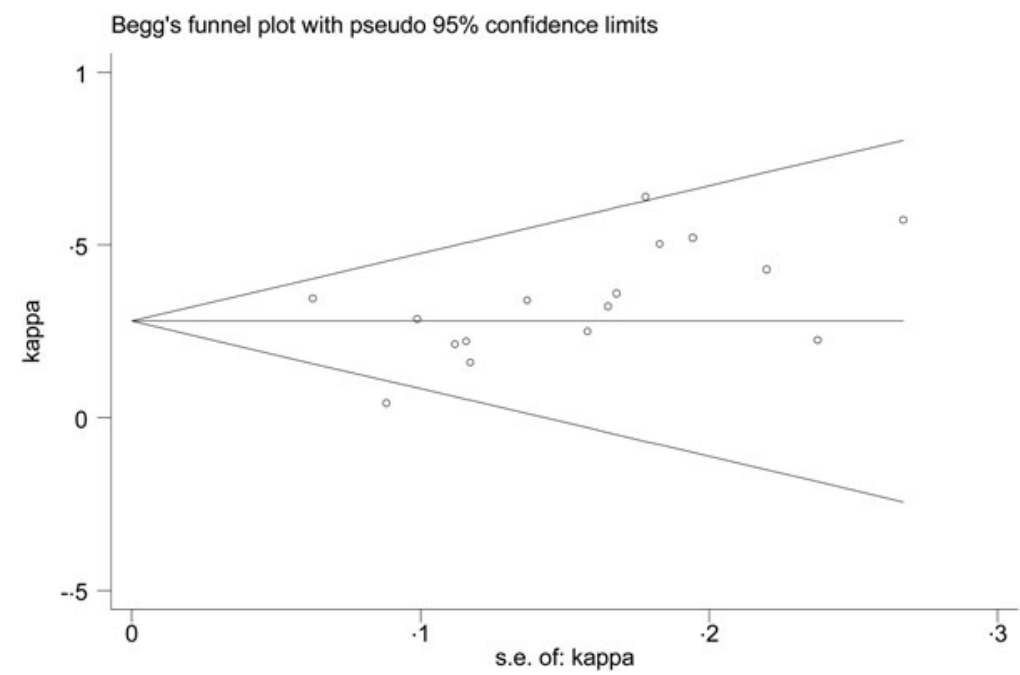

Fig. 2. Funnel plot of $\kappa$ estimate across the studies.

$0 \cdot 26(0 \cdot 19-0 \cdot 33)$, respectively. The $\kappa$ estimate for low quality and intermediate quality were $0.28(0.10$ $0 \cdot 37)$ and $0 \cdot 28(0 \cdot 20-0 \cdot 36)$, respectively. Stratified analysis by TB burden country showed that $\kappa$ estimate $(95 \%)$ equal to $0.23(0 \cdot 11-0 \cdot 34)$ for high TB burden countries and $0 \cdot 29(0 \cdot 22-0 \cdot 36)$ for low TB burden countries (Fig. 4a-d). Meta-regression plot showed that there was no difference in $\kappa$ estimate across different sample size and higher $\kappa$ estimate occurred at older ages (Fig. 5a and b).

\section{DISCUSSION}

Several meta-analyses have been reported pooled estimates for clinical agreement between IGRA and TST but not among HD patients. Our results showed only limited agreement between TST and QFT-GIT for the detection of LTBI in HD patients. Quality of studies had no significant effect on the results, though we identified no high-quality studies. Clinical agreement between the tests among BCG-vaccinated patients had lower point estimates, and although confidence bounds overlapped substantially. Low cut-off point of induration size in TST was associated with higher agreement. After summation of test results of the included study, it found that discordance of TST-l QFT-GIT+ was more than TST+/QFT-GIT-.

Relevant meta-analysis in HD patients was conducted by Ferguson et al. to evaluate diagnostic accuracy of tests for LTBI in HD patients [21]. The TST 


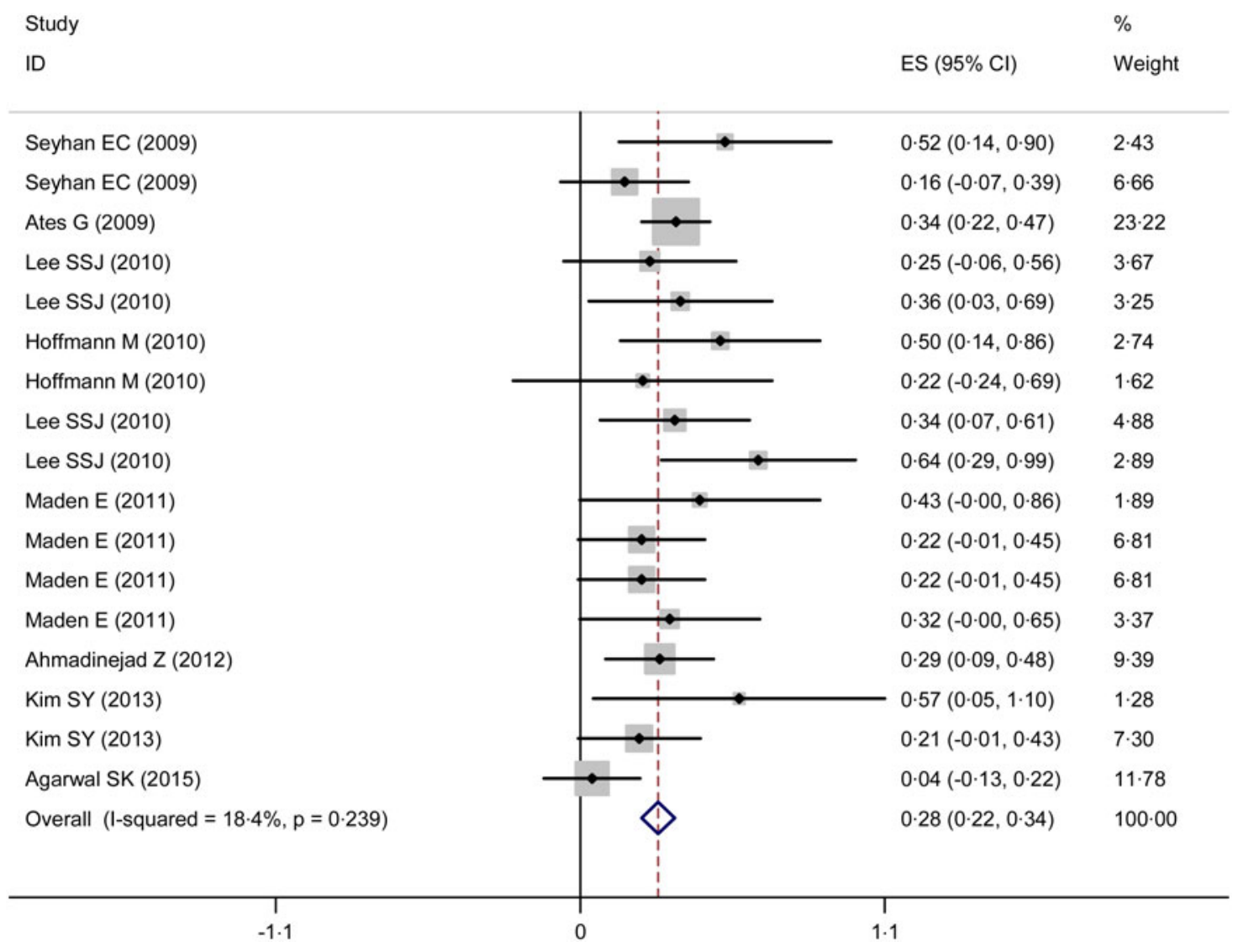

Fig. 3. Forest plot of $\kappa$ estimate across the studies.

had a pooled sensitivity of $31 \%$ and specificity of $63 \%$. In addition, the pooled sensitivity and specificity of QFT-GIT test were 53\% and $69 \%$, respectively. Their results display that QFT-GIT test was more sensitive than TST, but did not estimate amount of agreement between two tests.

We identified four previously reported metaanalyses comparing QFT-GIT and TST using $\kappa$ statistics. One focused on children [22], but did not provide pooled $\kappa$ estimates, and showed a variable range of reported agreement $(\kappa 0 \cdot 17-0 \cdot 86)$. The second studied agreement among high-risk contacts and reported fair-to-moderate agreement for $\kappa$ and prevalenceadjusted bias-adjusted $\kappa$ (PABAK), respectively [23]. They indicated that highest agreement is associated with TST cut-off point $\geqslant 15$, which is inconsistent with our results. Third meta-analysis assessed LTBI screening in immigrants to low-incidence countries [24]. Their results display a moderate concordance

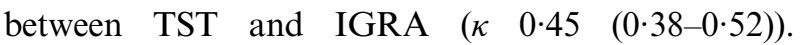
Another meta-analysis pooled $\kappa$ estimate in health care workers by Doosti-Irani et al. [25]. They found that agreement between the two tests estimated to be fair among health care workers, in addition, they found that $\kappa$ estimate for the TST cut-off point of $\geqslant 15 \mathrm{~mm}$ was more than other TST cut-off points. Among health care workers, BCG vaccinated individuals showed lower agreement between the two tests for diagnosis of LTBI [25].

Compared with TST, IGRAs have higher $M$. tuberculosis specificity, provide quick results and do not require subjective clinical measurement of induration size with the arising possibility of bias [26]. Although TST is cheaper and may be more costeffective [27, 28]. Uraemic state, long-term dialysis associated malnutrition, hyperparathyroidism and vitamin $\mathrm{D}$ deficiency cause stimulatory malfunction in antigen-presenting cells and prolong inflammatory response of monocytes which weakens cellular immunity [29-32]. Impaired cellular immunity may explain anergy and high rate of false-negative results of TST (lower sensitivity) in HD patients. Additionally, high risk of LTBI, particularly after kidney transplantation, confirms that a lower sensitivity 
(a)

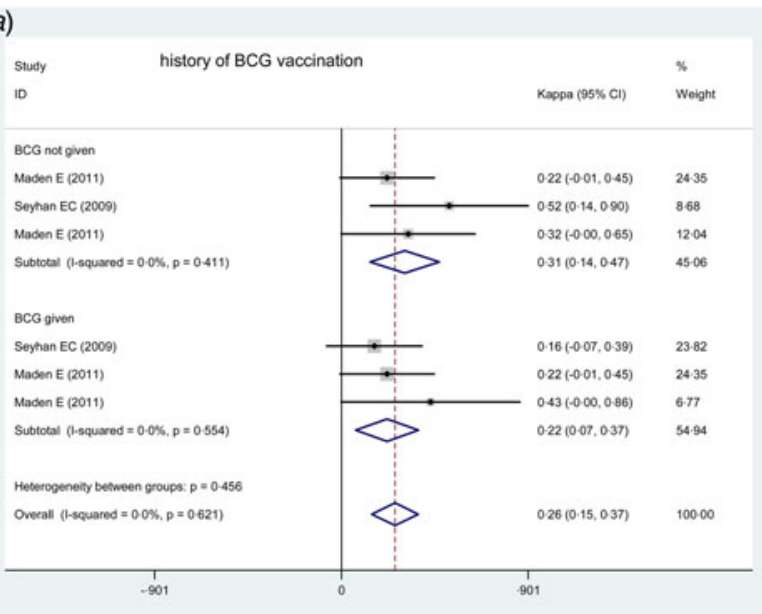

(c)

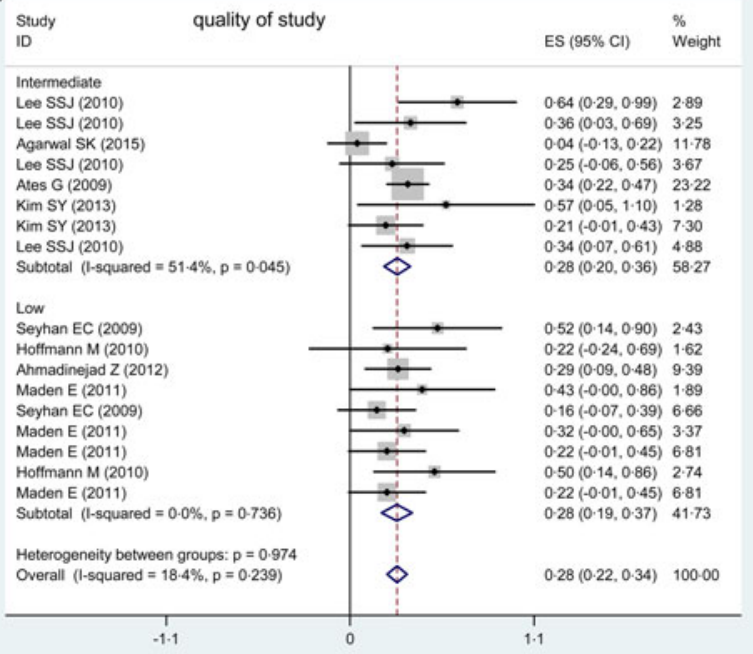

(b)

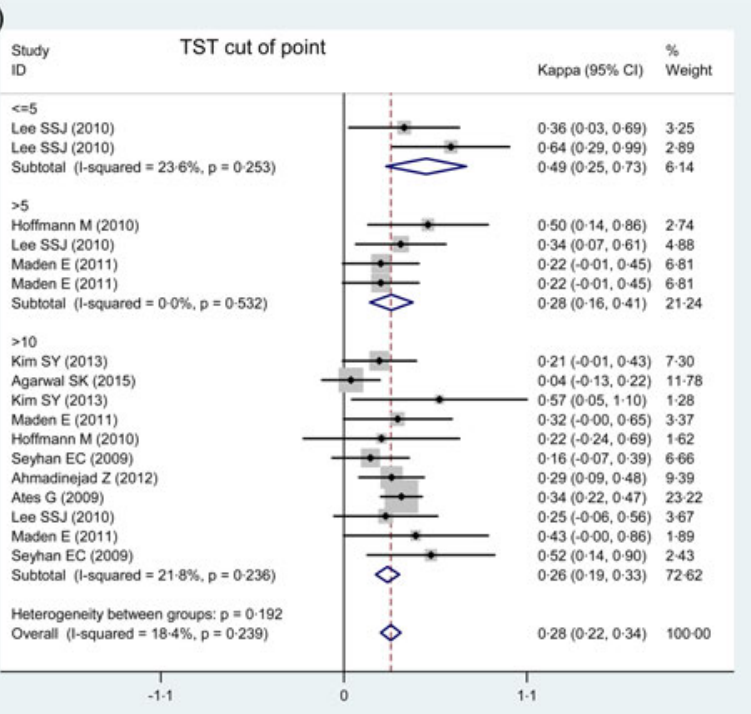

(d)

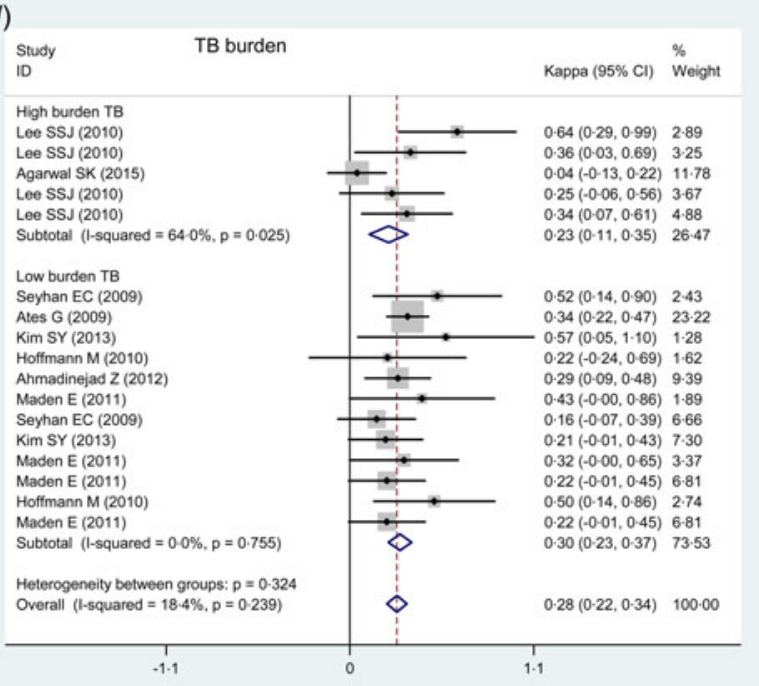

Fig. 4. Subgroup analysis of $\kappa$ estimate according to (a) history of BCG vaccination, (b) TST cut-off point, (c) quality of study and (d) TB burden.

may result in substantial clinical consequences [33]. A recent study of HD patients showed that QFT-GIT is the most cost-effective with compared with TST and chest X-ray (CXR) because of higher level of willingness-to-pay. Among HD patients, TST and CXR are more cost-effective only when the rate of BCG vaccination is $0 \cdot 18$ or lower [34].

Our study has limitations. We did not construct pooled PABAK values because the lack of information on TST-/QFT-GIT-, TST-/QFT-GIT+, TST +/QFT-GIT-, TST+/QFT-GIT+ meant we were unable to derive that statistic. However, prevalence of disease and bias in measurements are known to have significant effect on $\kappa$ estimate [35] as has been demonstrated in two previous meta-analyses [23, 25]. We pooled $\kappa$ estimates from countries with both high and low incidence of TB. Agreement between two tests may possibly be higher in very high or very low incidence countries. In addition, there was no high-quality study in our meta-analysis and half of included studies had low quality.

In conclusion, we detected limited agreement between TST and QFT-GIT for diagnosis of LTBI in HD patients. The agreement is lower still in BCG-vaccinated patients. According to the higher discordance of TST-/QFT-GIT+ in compared with TST +/QFT-GIT-, it seems QFT-GIT is superior to TST for detecting LTBI in HD patients. Our findings 

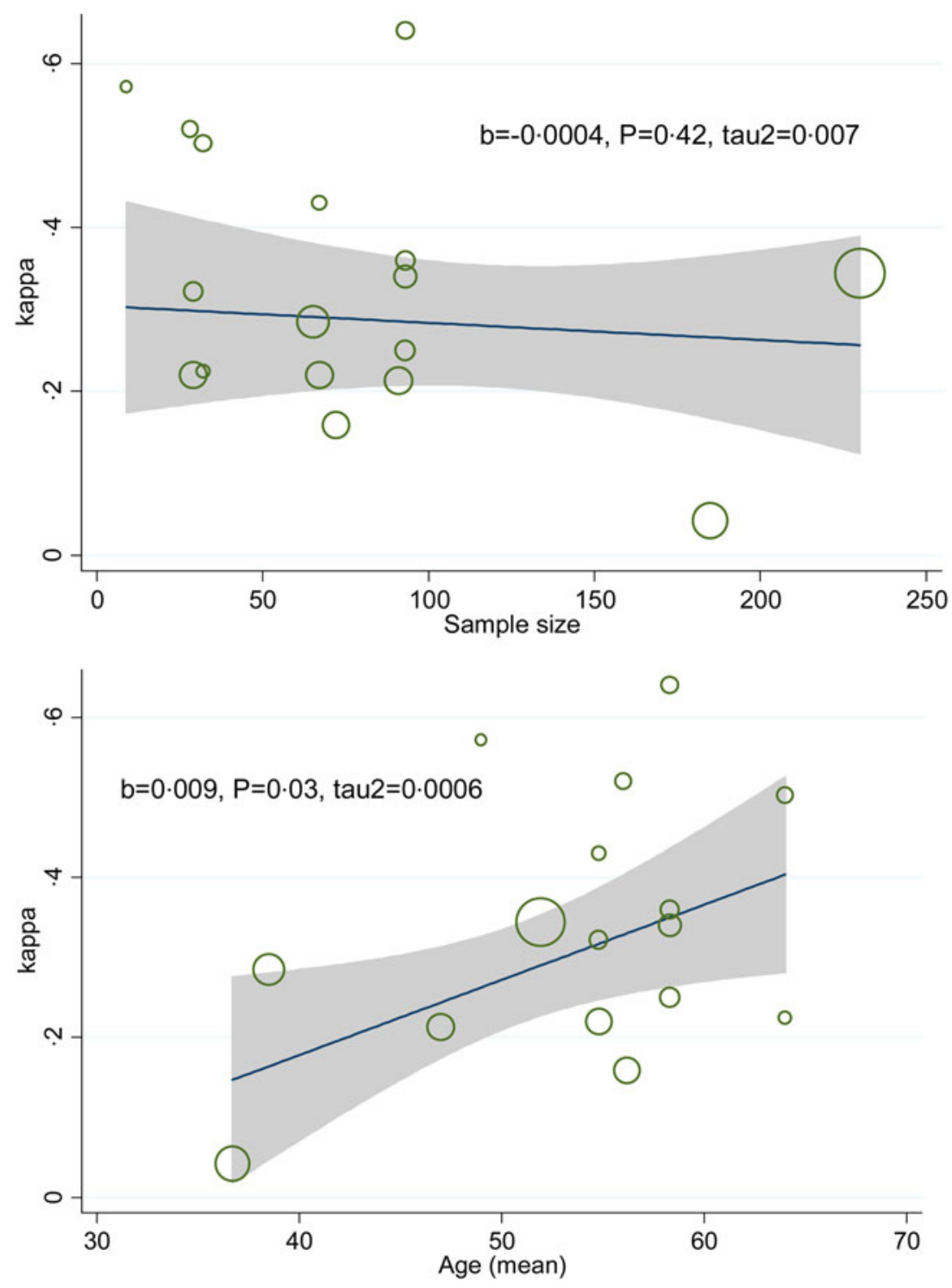

Fig. 5. Meta-regression plot of variation $\kappa$ by sample size and age.

suggest that TST and QFT-GIT are not interchangeable in HD patients and that QFT-GIT may be diagnostically superior for this vulnerable group of patients.

\section{SUPPLEMENTARY MATERIAL}

The supplementary material for this article can be found at https://doi.org/10.1017/S0950268817000334

\section{ACKNOWLEDGEMENTS}

The authors would like to thank statistics consultants of Research Development Center of Sina Hospital for their technical assistance.

\section{DECLARATION OF INTERESTS}

None.

\section{REFERENCES}

1. Chou KJ, et al. Tuberculosis in maintenance dialysis patients. Nephron 2001; 88: 138-143.

2. Moore DA, et al. High rates of tuberculosis in end-stage renal failure: the impact of international migration. Emerging Infectious Diseases 2002; 8: 77-78.

3. Kim SY, et al. Comparison of the tuberculin skin test and interferon-gamma release assay for the diagnosis of latent tuberculosis infection before kidney transplantation. Infection 2013; 41: 103-110.

4. Farhat M, et al. False-positive tuberculin skin tests: what is the absolute effect of BCG and non-tuberculous 
mycobacteria? International Journal of Tuberculosis and Lung Disease: the Official Journal of the International Union Against Tuberculosis and Lung Disease 2006; 10: 1192-1204.

5. Lee YM, et al. Risk factors for false-negative results of T-SPOT.TB and tuberculin skin test in extrapulmonary tuberculosis. Infection 2013; 41: 1089-1095.

6. World Health Organization. Use of tuberculosis interferon-gamma release assays (IGRAs) in low-and middle-income countries: policy statement. Geneva, 2011.

7. Diel R, et al. Interferon-gamma release assays for the diagnosis of latent Mycobacterium tuberculosis infection: a systematic review and meta-analysis. European Respiratory Journal 2011; 37: 88-99.

8. Diel R, et al. Predictive value of a whole blood IFNgamma assay for the development of active tuberculosis disease after recent infection with Mycobacterium tuberculosis. American Journal of Respiratory and Critical Care Medicine 2008; 177: 1164-1170.

9. Sester U, et al. T-cell activation follows Th1 rather than Th2 pattern in haemodialysis patients. Nephrology, Dialysis, Transplantation: Official Publication of the European Dialysis and Transplant Association European Renal Association 2000; 15: 1217-1223.

10. Chung WK, et al. Validity of interferon-gamma-release assays for the diagnosis of latent tuberculosis in haemodialysis patients. Clinical Microbiology and Infection: the Official Publication of the European Society of Clinical Microbiology and Infectious Diseases 2010; 16: 960-965.

11. Seyhan EC, et al. Comparison of the QuantiFERONTB Gold In-Tube test with the tuberculin skin test for detecting latent tuberculosis infection in hemodialysis patients. Transplant Infectious Disease: an Official Journal of the Transplantation Society 2010; 12: 98-105.

12. von Elm E, et al. Strengthening the Reporting of Observational Studies in Epidemiology (STROBE) statement: guidelines for reporting observational studies. BMJ (Clinical Research ed.) 2007; 335: 806-808.

13. Fleiss JL, Levin B, Paik MC. Statistical Methods for Rates and Proportions. New Jersey, USA: John Wiley \& Sons, 2013.

14. Agarwal SK, et al. Comparison of interferon gamma release assay \& tuberculin skin tests for diagnosis of latent tuberculosis in patients on maintenance haemodialysis. Indian Journal of Medical Research 2015; 141: 463-468.

15. Ahmadinejad Z, et al. Diagnosis of latent tuberculosis infection in candidates for kidney transplantation (comparison of two tests). Acta Medica Iranica 2012; 50: 305-310.

16. Ates $\mathbf{G}$, et al. Comparison of interferon-gamma release assay versus tuberculin skin test for latent tuberculosis screening in hemodialysis patients. Biotechnology \& Biotechnological Equipment 2009; 23: 1242-1246.

17. Hoffmann M, et al. Assessment of an Interferon-gamma release assay for the diagnosis of latent tuberculosis infection in haemodialysis patient. Swiss Medical Weekly 2010; 140(19-20): 286-292.
18. Jeong JC, et al. Utility of QuantiFERON-TB assay for prediction of tuberculosis development in kidney transplant patients in an intermediate-tuberculosis-burden country: lack of evidence for enhanced prediction for short-term tuberculosis development. Transplantation Proceedings 2014; 46: 583-587.

19. Lee SS, et al. High prevalence of latent tuberculosis infection in patients in end-stage renal disease on hemodialysis: comparison of QuantiFERON-TB GOLD, ELISPOT, and tuberculin skin test. Infection 2009; 37: 96-102.

20. Maden E, et al. Evaluation of performance of quantiferon assay and tuberculin skin test in end stage renal disease patients receiving hemodialysis. New Microbiologica 2011; 34: 351-356.

21. Ferguson TW, et al. The diagnostic accuracy of tests for latent tuberculosis infection in hemodialysis patients: a systematic review and meta-analysis. Transplantation 2015; 99: 1084-1091.

22. Machingaidze S, et al. The utility of an interferon gamma release assay for diagnosis of latent tuberculosis infection and disease in children: a systematic review and meta-analysis. Pediatric Infectious Disease Journal 2011; 30: 694-700.

23. Ayubi E, et al. The clinical usefulness of tuberculin skin test versus interferon-gamma release assays for diagnosis of latent tuberculosis in HIV patients: a metaanalysis. PLoS ONE 2016; 11: e0161983.

24. Campbell JR, et al. Latent tuberculosis infection screening in immigrants to low-incidence countries: a meta-analysis. Molecular Diagnosis \& Therapy 2015; 19: 107-117.

25. Doosti-Irani A, Ayubi E, Mostafavi E. Tuberculin and QuantiFERON-TB-Gold tests for latent tuberculosis: a meta-analysis. Occupational Medicine (Oxford, England) 2016; 66: 437-445.

26. Mazurek GH, et al. Updated guidelines for using interferon gamma release assays to detect Mycobacterium tuberculosis infection - United States, 2010. MMWR Recommendations and Reports: Morbidity and Mortality Weekly Report Recommendations and Reports 2010; 59: $1-25$.

27. Anon. KDIGO clinical practice guideline for the care of kidney transplant recipients. American Journal of Transplantation: Official Journal of the American Society of Transplantation and the American Society of Transplant Surgeons 2009; 9 (Suppl 3): S1-155.

28. Chadban SJ, et al. KHA-CARI guideline: KHA-CARI adaptation of the KDIGO Clinical Practice Guideline for the Care of Kidney Transplant Recipients. Nephrology (Carlton, Vic) 2012; 17: 204-214.

29. Chan T. Vitamin D deficiency and susceptibility to tuberculosis. Calcified Tissue International 2000; 66: 476-478.

30. Girndt $\mathbf{M}$, et al. Impaired cellular immune function in patients with end-stage renal failure. Nephrology Dialysis Transplantation 1999; 14: 2807-2810.

31. Smirnoff M, et al. Tuberculin and anergy skin testing of patients receiving long-term hemodialysis. Chest 1998; 113: $25-27$. 
32. Tzanno-Martins C, et al. Restoration of impaired T-cell proliferation after parathyroidectomy in hemodialysis patients. Nephron 2000; 84: 224-227.

33. Chia S, et al. Risk of tuberculosis in dialysis patients: a population-based study. International Journal of Tuberculosis and Lung Disease 1998; 2: 989-991.
34. Kowada A. Cost effectiveness of the interferon- $\gamma$ release assay for tuberculosis screening of hemodialysis patients. Nephrology Dialysis Transplantation 2013; 28: 682-688.

35. Sim J, Wright CC. The kappa statistic in reliability studies: use, interpretation, and sample size requirements. Physical Therapy 2005; 85: 257-268. 